\title{
Treatment of prostatic stromal sarcoma with robot-assisted laparoscopic radical prostatectomy in a young adult: A case report
}

\author{
QI-QI MAO, SHUO WANG, PING WANG, JIE QIN, DAN XIA and LI-PING XIE \\ Department of Urology, The First Affiliated Hospital, School of Medicine, \\ Zhejiang University, Hangzhou, Zhejiang 310003, P.R. China
}

Received December 7, 2014; Accepted January 21, 2016

DOI: $10.3892 / \mathrm{ol} .2016 .4249$

\begin{abstract}
The present study reports a rare case of prostatic stromal sarcoma (PSS) treated with a robot-assisted laparoscopic radical prostatectomy (RLRP). A 32-year-old man presented to the Department of Urology, The First Affiliated Hospital, School of Medicine, Zhejiang University (Hangzhou, China) with obstructive voiding symptoms that had persisted for 2 years. A computed tomography scan of the pelvis revealed an 8-cm prostatic mass protruding into the bladder. A transperineal ultrasound-guided prostate biopsy revealed a diagnosis of PSS. An RLRP was performed, and neither chemotherapy nor radiation therapy were administered prior to or subsequent to the surgery. No recurrence of the tumor was indicated at 6 months post-surgery. To the best of our knowledge, $\leq 30$ cases of PSS have been reported in the English literature, and the present study is only the second case to be treated with RLRP.
\end{abstract}

\section{Introduction}

Prostatic stromal sarcoma (PSS) is an extremely rare type of adult non-epithelial malignant tumor affecting the prostate. In 1998, Gaudin et al (1) classified PSS into two categories: Prostatic stromal proliferation of uncertain malignant potential and PSS. The etiology and pathogenesis of PSS is currently unknown, and no confirmed risk factors have been identified $(1,2)$. As the majority of patients present with obstructive urinary symptoms, the diagnosis of prostatic stromal sarcoma is frequently made following open prostatectomy or transurethral resection of the prostate (3). Owing to the rarity of the tumor, with $<30$ cases reported in the literature, the optimal treatment for this disease has not yet been determined (3). The robot-assisted laparoscopic radical prostatectomy (RLRP) has grown increasingly popular and rapidly equated itself as the

Correspondence to: Professor Shuo Wang, Department of Urology, The First Affiliated Hospital, School of Medicine, Zhejiang University, 79 Qingchun Road, Hangzhou, Zhejiang 310003, P.R. China

E-mail: urowang@126.com

Key words: prostatectomy, robotics, sarcoma most frequently used modality to treat organ-confined prostate cancer (4). A limited number of PSS cases have been treated with RLRP due to the low prevalence and poor prognosis of such malignancies. The current study presents the case of a patient with PSS who was treated with RLRP.

\section{Case report}

On April 8, 2014, a 32-year-old man was referred to the Department of Urology, The First Affiliated Hospital, School of Medicine, Zhejiang University (Hangzhou, China) with progressive obstructive voiding symptoms that had persisted for 2 years. The patient had also developed acute urinary retention that had been apparent for 3 days. A digital rectal examination revealed a huge solid mass. Serum levels of prostate-specific antigen (PSA) were not elevated $(0.44 \mathrm{ng} / \mathrm{ml}$; normal range, 0-4 $\mathrm{ng} / \mathrm{ml}$ ). A computed tomography scan (SOMATOM Definition AS20; Siemens AG, Munich, Germany) of the pelvis showed an $8 \times 6-\mathrm{cm}$ prostatic mass protruding into the bladder (Fig. 1A). A magnetic resonance imaging scan (Signa HDxt 3.0T; GE Healthcare Life Sciences, Chalfont, UK) revealed a multinodular mass, with homogeneous low signal intensity on T1-weighted imaging (Fig. 1B) and heterogeneous high signal intensity on T2-weighted imaging (Fig. 1C). No enlarged lymph nodes were detected. A transperineal, ultrasound-guided prostate biopsy was performed on April 16, 2014. The histopathological examination revealed spindle tumor cells with cytologic atypia and mitoses, thus resulting in a diagnosis of PSS. On May 14, 2014, the patient underwent RLRP without pelvic lymphadenectomy.

For the RLRP, the patient was placed in the dorsal lithotomy position with his arms secured to his side. A pneumoperitoneum was created using a VERESS pneumoperitoneum needle (Karl Storz GmbH \& Co. KG, Tuttlingen, Germany). The trocars were positioned with similar placement to a standard robotic prostatectomy: A 12-mm camera port was placed $2 \mathrm{~cm}$ cephalad to the umbilicus; two 8-mm metal robotic ports were placed bilaterally along the midclavicular line at the level of the umbilicus; an accessory 5-mm port for suction was placed lateral and superior to the camera port; a second 12-mm assistant port was placed $8-10 \mathrm{~cm}$ lateral to the right robotic port; and a third $8-\mathrm{mm}$ robotic port was placed $8 \mathrm{~cm}$ lateral to the left robotic port, $2 \mathrm{~cm}$ above and anteriorly to the anterior superior iliac spine. The table was moved to a deep Trendelenburg position. 
A

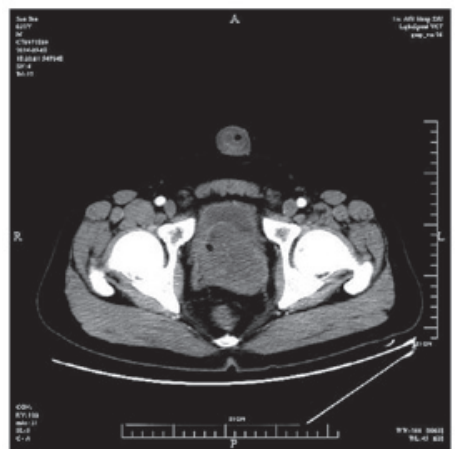

B

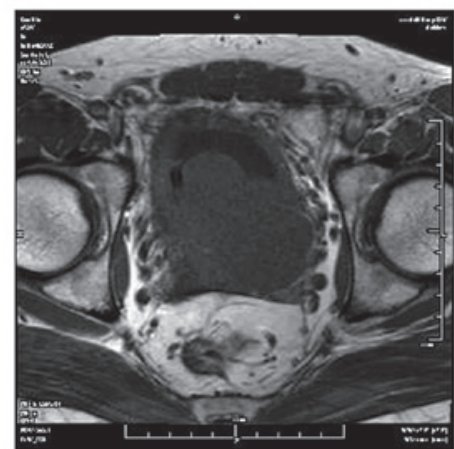

C

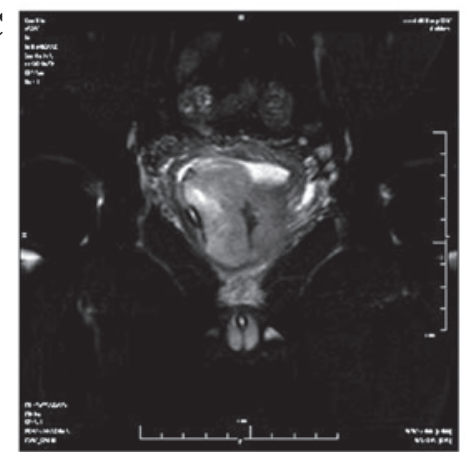

Figure 1. (A) Pelvic computed tomography showing a prostatic non-homogenous mass lesion protruding into the bladder space. (B) T1-weighted magnetic resonance imaging showing a homogeneous low signal intensity mass. (C) T2-weighted imaging showing a multinodular mass with heterogeneous high signal intensity.

A

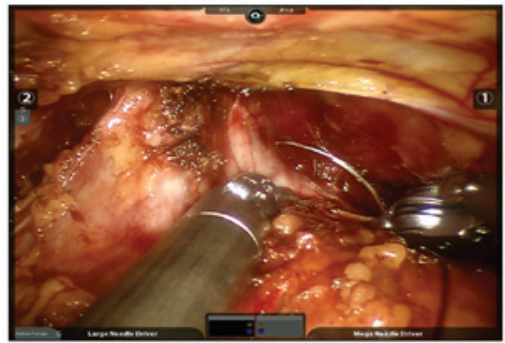

C

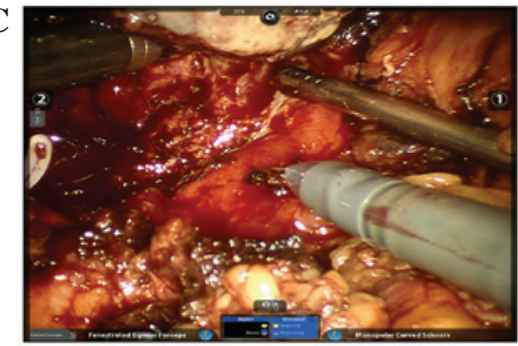

B

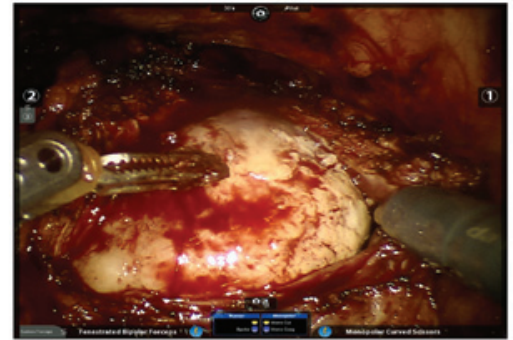

D

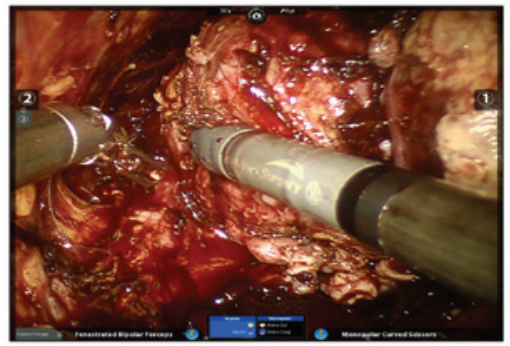

Figure 2. (A) Ligation of the dorsal vein plexus using 2-0 Vicryl sutures. (B) Wide incision of the anterior bladder wall to fully expose the tumor protruding into the bladder. (C) Dissection of the posterior bladder neck following elevation of the tumor. (D) Careful dissection of the lateral pedicle prior to posterior dissection.

A

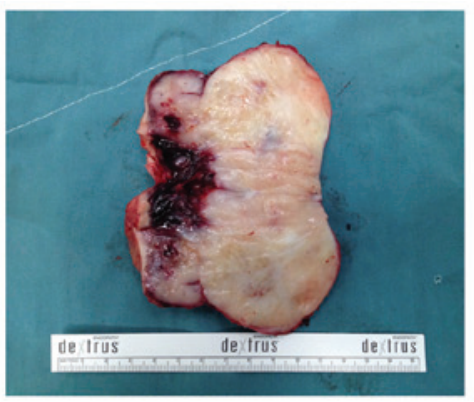

B

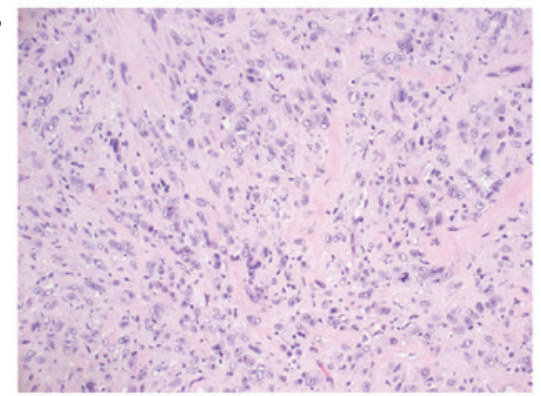

C

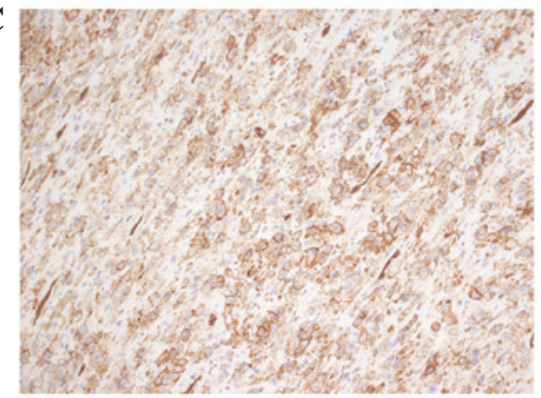

Figure 3. (A) Gross appearance of the prostatectomy specimen, exhibiting a whitish-yellow multinodular appearance with focal necrosis. (B) Tumor cells showing a spindled morphology, with pleomorphic nuclei and numerous atypical mitoses (hematoxylin and eosin stain; magnification, $\mathrm{x} 200$ ). (C) Immunostaining showing the expression of cluster of differentiation 34 in the tumor cells (magnification, x200).

The da Vinci robotic system (Intuitive Surgical Inc., Sunnyvale, CA, USA) was brought between the patient's legs, and the four arms were connected to the corresponding ports.

From a technical standpoint, the surgery was extremely challenging. The anterior prostatic surface and the endopelvic fascia were difficult to expose due to the lack of space. Control of the dorsal vein plexus was achieved by a figure-of-eight ligation, using 2-0 Vicryl sutures (Ethicon; Johnson \& Johnson,
New Brunswick, NJ, USA), with the advantage of flexibility (Fig. 2A). Considering that the preservation of the bladder neck is impossible and that the plane between the prostate and bladder neck could not be identified, the bladder was directly and widely incised to fully expose the mass protruding into the bladder (Fig. 2B). The bladder neck dissection was initiated bilaterally along the tumor. However, the dissection of the posterior bladder neck was challenging due to the poor elevation 
of the prostate. The third arm was used to lift the mass and gain increased elevation (Fig. 2C). This challenge became more significant during the posterior dissection for the marked adhesion between the rectum and the prostate. In order to minimize the risk of rectal injury, the dissection of the apical and lateral pedicles was performed first and progressed toward the midline (Fig. 2D). Following the removal of the tumor, the large bladder neck was reconstructed using the tennis racket technique with a 2-0 Vicryl suture in running fashion. The wide gap between the bladder and urethra that was left following the removal of the huge mass resulted in tension on the anastomosis, which was reduced by additional mobilization of the bladder, decreased Trendelenberg tilt and perineal pressure.

The patient recovered uneventfully post-operatively, and was discharged 2 weeks after the surgery. The resected specimen was a yellow-white tumor of $8 \mathrm{~cm}$ in diameter, with occasional hemorrhagic foci. A pathological examination of the specimen revealed a PSS with negative surgical margins. Formalin-fixed, paraffin-embedded, 5- $\mu \mathrm{m}$ thick sections were immunohistochemically diffusely positive for cluster of differentiation (CD34; anti-mouse antibody; catalog no., 14-0341-85; dilution, 1:250; eBioscience, Inc., San Diego, CA, USA), and focally positive for B-cell lymphoma 2, but not for CD117, vimentin, epithelial membrane antigen and progesterone receptor. No recurrence or distant metastasis of the tumor has occurred following the surgical intervention, and the patient continues to be followed up.

\section{Discussion}

Prostate sarcoma is a rare malignancy that is associated with a poorer prognosis compared with prostate cancer. PSS is an extremely rare subtype of prostate sarcoma with $<30$ documented cases $(2,3,5,6)$. The majority of these lesions present in the sixth and seventh decades of life, and the majority of patients present with symptoms of urethral obstruction, such as in the present case $(2,3,5,6)$. Patients with PSS usually have a PSA level within the normal range. Histologically, the tumor cells have an ovoid, spindle shape, with pleomorphic nuclei. CD34, CD117, vimentin and progesterone receptor may assist in distinguishing between these tumors and other prostatic mesenchymal neoplasms, for example, rhabdomyosarcoma and leiomyosarcoma are negative for CD34 and positive for vimentin (7).

PSS presents a significant therapeutic challenge. Due to the rare occurrence of PSS and the paucity of published literature, the optimal treatment for the disease is unknown. Osaki et al (2) presented a case of PSS in which a suprapubital radical prostatectomy was performed without adjuvant therapy, with no recurrence reported at 8 years post-surgery. Reese et al (3) applied an aggressive multimodality approach in the management of a PSS, including neoadjuvant chemotherapy and radiation, which resulted in a complete response in the primary lesion, followed by radical cystoprostatectomy. Chang et al (8) reported one case of PSS treated with radical cystoprostatectomy followed by radiotherapy, and the patient was alive and well 5 months after treatment. The outcomes of these studies are challenging to interpret due to the heterogeneity of treatment modalities. However, a complete radical surgical resection (radical prostatectomy or cystoprostatectomy) remains the preferred treatment that is most likely to result in long-term survival (1). In the present study, with consideration to the particularly young age of the patient, and due to no apparent bladder invasion on the pre-operative images, the decision was made to perform a radical prostatectomy with the expectation of sparing the bladder.

Open surgery, the standard laparoscopic technique and the robot-assisted technique are surgical options for the management of PSS. Previously, the feasibility of laparoscopic surgery for PSS has not been reported. To the best of our knowledge, only the study by Choi et al (9) has previously reported on the experience of conducting a robot-assisted excision of a PSS, in 2014. RLRP has become a common, widely accepted and effective surgical choice for patients with prostate cancer. In the present case, the traditional laparoscopic instruments were limited by the narrow manipulation space in the deep pelvis. The da Vinci robot-assisted laparoscopic surgical system possesses various advantages over standard laparoscopic surgery $(10,11)$. The da Vinci system generates an accurate three-dimensional depth of field using a high resolution, and the third arm maintains a strong retraction for exposure, thus enabling the surgeon to acquire a visual field $(10,11)$. Furthermore, the da Vinci system offers a high degree of freedom for operating the instruments, and achieves the separation and dissection of tumors with more precision, while minimizing the possibility of intraoperative complications, including ureteral and rectal injury $(10,11)$.

PSS is an aggressive disease with a poor prognosis. Despite the challenges that were experienced due to the large tumor size and adhesions, the en bloc resection of the tumor with negative margins and favorable short-term results observed in the present case demonstrate that robotic extirpation is a feasible and effective option for the management of PSS.

\section{References}

1. Gaudin PB, Rosai J and Epstein JI: Sarcomas and related proliferative lesions of specialized prostatic stroma: A clinicopathologic study of 22 cases. Am J Surg Pathol 22: 148-162, 1998.

2. Osaki M, Osaki M, Takahashi C, Miyagawa T, Adachi H and Ito H: Prostatic stromal sarcoma: Case report and review of the literature. Pathol Int 53: 407-411, 2003.

3. Reese AC, Ball MW, Efron JE, Chang A, Meyer C and Bivalacqua TJ: Favorable response to neoadjuvant chemotherapy and radiation in a patient with prostatic stromal sarcoma. J Clin Oncol 30: e353-e355, 2012.

4. Wirth MP and Froehner M: Robot-assisted radical prostatectomy: The new gold standard? Eur Urol 57: 750-751, 2010.

5. Tamada T, Sone T, Miyaji Y, Kozuka Y and Ito K: MRI appearance of prostatic stromal sarcoma in a young adult. Korean J Radiol 12: 519-523, 2011.

6. Yamazaki H, Ohyama T, Tsuboi T, Taoka Y, Kohguchi D, Iguchi $\mathrm{H}$ and Ao T: Prostatic stromal sarcoma with neuroectodermal differentiation. Diagn Pathol 7: 173, 2012.

7. Westra WH, Gerald WL and Rosai J: Solitary fibrous tumor. Consistent CD34 immunoreactivity and occurrence in the orbit. Am J Surg Pathol 18: 992-998, 1994.

8. Chang YS, Chuang CK, Ng KF and Liao SK: Prostatic stromal sarcoma in a young adult: A case report. Arch Androl 51: 419-424, 2005

9. Choi SH, Kim TH, Yoon GS, Chung SK, Kim BW and Kwon TG: Two different surgical approaches for prostatic stromal sarcoma: Robot-assisted laparoscopic radical prostatectomy and open radical cysto-prostatectomy with ileal conduit. Korean J Urol 55: 620-623, 2014.

10. Trabulsi EJ,Zola JC, Colon-Herdman A,Heckman JE, Gomella LG and Lallas CD: Minimally invasive radical prostatectomy: Transition from pure laparoscopic to robotic-assisted radical prostatectomy. Arch Esp Urol 64: 823-829, 2011 (In Spanish).

11. Finkelstein J, Eckersberger E, Sadri H, Taneja SS, Lepor H and Djavan B: Open versus laparoscopic versus robot-assisted laparoscopic prostatectomy: The European and US experience. Rev Urol 12: 35-43, 2010. 